\title{
Relevance and implementation of patient- specific dosimetry in targeted radionuclide therapy
}

\author{
Manuel Bardiès ${ }^{1,2}$ \\ ${ }^{I}$ Centre de Recherches en Cancérologie de Toulouse, Toulouse, France \\ ${ }^{2}$ UMR 1037, INSERM, Université Toulouse III Paul Sabatier, Toulouse, France
}

\section{Introduction}

Targeted Radionuclide Therapy (TRT) also called sometimes Molecular Radiotherapy (MRT) is dealing with the therapeutic applications of nuclear medicine. TRT aims at selectively irradiate targets (usually tumours) by the means of a biologic vector labelled with a radioactive isotope. The specificity of the therapy is achieved by the targeting vector, whereas low range radiation irradiates in the neighbourhood of the emission point, thereby insuring selective irradiation of the target and reduced irradiation of surrounding tissues.

According to the vector, one can define TRT specialities:

- Radioimmunotherapy (RIT) uses monoclonal antibodies directed against tumour specific antigens.

- Peptide Receptor Radionuclide Therapy (PRRT) is using peptides directed against some receptors expressed by the tumour cell (e.g. somatostatine receptors in the case of the recently approved Lutathera).

- Metabolic Radiotherapy is a general term to define all situations where the vector participates to the metabolism of the tumour. The therapy with iodine 131 or thyroid pathologies enters in that category. Bone palliation with bone seeking agents also enters into that category.

Selective Internal Radiotherapy (SIRT) of liver tumours with ${ }^{90} \mathrm{Y}$ microspheres does not strictly speaking belongs to TRT, as radioactive microspheres are medical devices administered via hepatic arteries and not radiopharmaceuticals.

The choice of the isotope has been a long matter of debate - and flourishing research - to identify "the" ideal candidate [1]. The perfect isotope should emit low range radiation, in order to preserve the selectivity achieved by the vector. Beta minus emitters with a maximum range between some $\mathrm{mm}$ fraction to $1 \mathrm{~cm}$ enter in that category. The possible emission of gamma in a range that allows scintigraphic imaging (around $150 \mathrm{keV}$ ) and with a rather low yield to avoid non-selective irradiation is a bonus. In addition, the physical half-life of the emitter should be adapted to the biological half-life of the vector, in order to avoid either the premature decay of the radioactive sources, or the premature elimination of radioactive compound. Some commonly used isotopes for TRT include ${ }^{131} \mathrm{I},{ }^{90} \mathrm{Y},{ }^{177} \mathrm{Lu}$, ${ }^{153} \mathrm{Sm}$. Alpha emitters have also been proposed, but their short range would normally restrict their use to small and easily (quickly) accessible targets, with the notable exception of ${ }^{223} \mathrm{Ra}$ that received FDA/EMA approval for the treatment of bone metastases. 


\section{Dosimetry of Targeted Radionuclide Therapy}

\subsection{Relevance of clinical dosimetry for TRT}

TRT is usually administered as a "radioactive chemotherapy", where patients are being given the same amount of drug (activity) in a "one size fits all" approach [2,3]. Activity administration (in $\mathrm{Bq}$, or $\mathrm{Bq} / \mathrm{kg}$ ) is defined after "dose" escalation (phase I) clinical trials that assess the toxicity of the drug. However, since the pharmacokinetics of a radiolabelled compound varies from a patient to another, administering to all the activity that induces toxicity to the most fragile will undertreat a large fraction of patients. This calls for patient-specific administrations of the radiolabelled compound, which can be envisaged when it is possible thanks to gamma emissions to follow the fate of the radiolabelled vector in the patient.

The biological effect of procedures involving ionizing radiation can usually be evaluated in the light of the energy delivered to the tissues. The absorbed dose (energy/unit mass, in $\mathrm{J} / \mathrm{kg}$ or Gy) is an objective index of the irradiation pattern.

\subsection{Dosimetry formalism for TRT}

According to the MIRD formalism [4], $\bar{D}_{k}$, the absorbed dose delivered to a target $\mathrm{k}$ from radioactive sources in source regions $h$ can be computed as:

$$
\bar{D}_{k}=\sum_{h} \tilde{A}_{h} \times S_{(k \leftarrow h)}
$$

Where $\tilde{A}_{h}$ is the total number of decay (cumulated activity) in the source $\mathrm{h}$ during the irradiation process (in Bq.s), $S_{(k \leftarrow h)}$ is the $\mathrm{S}$ factor, or $\mathrm{S}$ value, i.e. the absorbed dose per unit cumulated activity, in Gy. Bq ${ }^{-1} \cdot \mathrm{s}^{-1}$. This simplified equation underlines the prerequisites for absorbed dose calculation determination: knowing the number of radioactive sources, within the patient as a function of time and integrating the time-activity curve for all region sources; implementing radiation transport to assess how emitted energy is deposited in surrounding media.

Clinical dosimetry is a chain of operations, each of which impacts the final result (Figure 1).

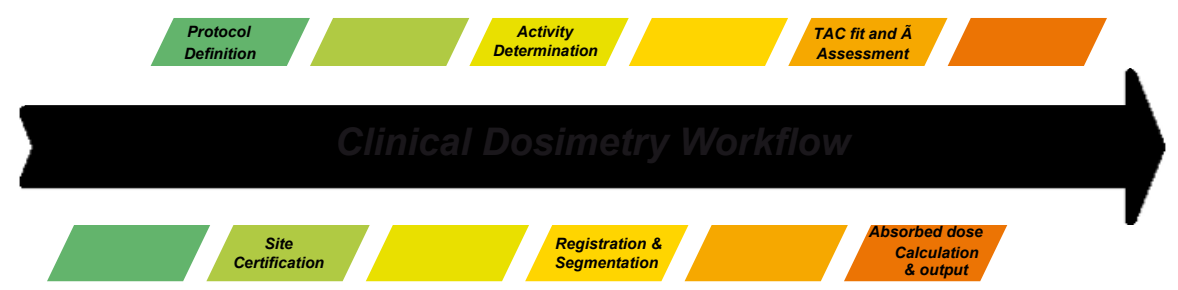


Figure 1: Clinical dosimetry workflow

The relevance of dosimetry in TRT relies on the assumption that an absorbed dose - effect relationship (ADER) can be put in evidence and used to monitor and optimise therapy.

In 2014 the EANM Dosimetry Committee reviewed published ADER evidences [5]. Conclusions were that ADER can be found whenever they are looked for using a relevant/adapted approach. That article also highlighted the fact that absorbed dose calculation is only one side of the ADER. The definition of the biological/clinical end-point is also an essential part of the clinical dosimetry protocol.

\subsection{Absorbed dose - effect relationships example}

For a given clinical endpoint, the way the dosimetry protocol is implemented will impact the conclusion. A very interesting example is given in the context of PRRT in the article of Barone et al. [6]. The study considered a subset of patients with neuroendocrine tumours, enrolled in a phase 1 multicentric study intended to establish the maximum tolerated doses of ${ }^{90} \mathrm{Y}$-DOTATOC in patients with metastatic, somatostatin-receptor positive tumours. That subset of 18 patients benefited from a dosimetric study, in order to evaluate the absorbed doses delivered to the kidneys during a pre-therapeutic administration of ${ }^{86}$ Y-DOTATOC ( 3 time-points). The therapy was delivered in 1 to 5 cycles. Therapeutic activity was modulated so that no patient received more than 27 Gy to the kidneys. Kidney toxicity was assessed by monitoring kidney function (serum creatinine and creatinine clearance).

PET quantitative imaging with ${ }^{86} \mathrm{Y}$ was challenging and eventually led to a specific publication[ix].

- Absorbed doses were initially computed using MIRDOSE 3.1 [7], under the assumption that all patients had the same kidney volume (but differentiating between male and female patients).

- A second (retrospective) study considered the actual volume of each patient's kidney, and rescaled the S values obtained from MIRD pamphlet 19 [8] to a patient-specific S value (and considered the absorbed dose delivered to the cortex rather than that to the whole kidney).

- A third approach included radiobiological parameters (from EBRT) to derive the Biologic Effective Dose (BED) delivered to the kidney cortex.

The study to the correlation between the loss of kidney function (toxicity) and either the kidney absorbed dose or BED was presented Barone et al. It clearly demonstrates how the dosimetric protocol impacts ADER.

In addition, from a dosimetric point of view, it also highlights how ALL aspects that lead from activity quantification to absorbed dose (or BED) matter to put in evidence ADERs: even a very refined activity determination (based on quantitative ${ }^{86}$ Y-DOTATOC PET imaging) will not provide good ADER if the same $\mathrm{S}$ value is used for all patients...

\section{Conclusions}

Further analysis of published evidence shows that there is no and there won't be a generic clinical dosimetry procedure. "Dosimetry that works", i.e. providing for ADERs is disease/application/radiopharmaceutical specific. 
The fact that there is no reference clinical dosimetry protocol generic enough to be adapted to all therapeutic applications of nuclear medicine should not prevent us from implementing what seems relevant at a given moment, using available resources. Documenting the protocol is an essential part of the reporting and should be taken as seriously as possible [9].

\section{References}

[1] Mausner LF, and Srivastava SC. Selection of radionuclides for radioimmunotherapy. Medical Physics. 1993;20(2).

[2] Chiesa C, Sjogreen Gleisner K, Flux G, Gear J, Walrand S, Bacher K, Eberlein U, Visser EP, Chouin N, Ljungberg M, Bardiès M, Lassmann M, Strigari L, and Konijnenberg MW. The conflict between treatment optimization and registration of radiopharmaceuticals with fixed activity posology in oncological nuclear medicine therapy. Eur J Nucl Med Mol Imaging. 2017;44(11): 1783-1786.

[3] Flux G, Sjogreen Gleisner K, Chiesa C, Lassmann M, Chouin N, Gear J, Bardiès M, Walrand S, Bacher K, Eberlein U, Ljungberg M, Strigari L, Visser E, Konijnenberg M. From fixed activities to personalized treatments in radionuclide therapy: lost in translation? Eur J Nucl Med Mol Imaging. 2018; 45(1):152-154.

[4] Loevinger R, Budinger TF, Watson EE. MIRD Primer for Absorbed Dose Calculations. Revised ed. New York, NY: The Society of Nuclear Medicine; 1991.

[5] Strigari L, Konijnenberg M, Chiesa C, Bardiès M, Du Y, Gleisner KS, Lassmann M, and Flux G. The evidence base for the use of internal dosimetry in the clinical practice of molecular radiotherapy. Eur J Nucl Med Mol Imaging. 2014;41(10):1976-88.

[6] Barone R, Borson-Chazot F, Valkema R, Walrand S, Chauvin F, Gogou L, Kvols LK, Krenning EP, Jamar F, and Pauwels S. Patient-specific dosimetry in predicting renal toxicity with 90Y-DOTATOC: relevance of kidney volume and dose rate in finding a doseeffect relationship. Journal of Nuclear Medicine. 2005;46(1 suppl):99S.

[7] Stabin MG. MIRDOSE: personal computer software for internal dose assessment in nuclear medicine. J Nucl Med. 1996;37:538 -546.

[8] Bouchet LG, Bolch WE, Blanco HP, Wessels BW, Siegel JA, Rajon DA, Clairand I, and Sgouros G. MIRD Pamphlet No 19: absorbed fractions and radionuclide S values for six age-dependent multiregion models of the kidney. J Nucl Med. 2003;44(7):1113-47.

[9] Lassmann M, Chiesa C, Flux G, Bardiès M, and EANM Dosimetry Committee. EANM Dosimetry Committee guidance document: good practice of clinical dosimetry reporting. Eur J Nucl Med Mol Imaging. 2011;38(1):192-200. 